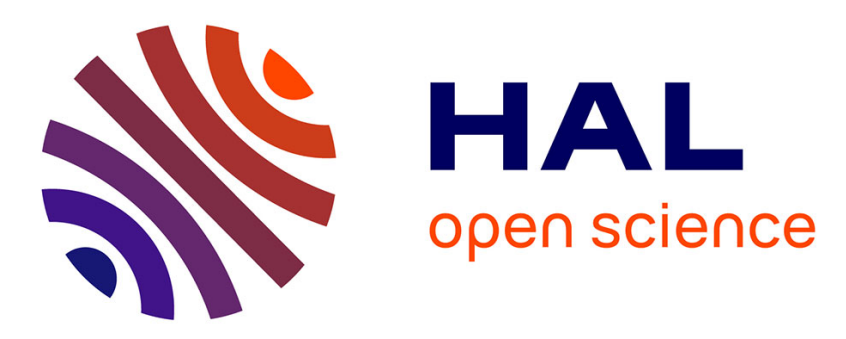

\title{
Spectral diffusion decay of spontaneous echoes in disordered systems
}

R. Maynard, R. Rammal, R. Suchail

\section{To cite this version:}

R. Maynard, R. Rammal, R. Suchail. Spectral diffusion decay of spontaneous echoes in disordered systems. Journal de Physique Lettres, 1980, 41 (12), pp.291-294. 10.1051/jphyslet:019800041012029100 . jpa-00231782

\section{HAL Id: jpa-00231782 https://hal.science/jpa-00231782}

Submitted on 1 Jan 1980

HAL is a multi-disciplinary open access archive for the deposit and dissemination of scientific research documents, whether they are published or not. The documents may come from teaching and research institutions in France or abroad, or from public or private research centers.
L'archive ouverte pluridisciplinaire $\mathbf{H A L}$, est destinée au dépôt et à la diffusion de documents scientifiques de niveau recherche, publiés ou non, émanant des établissements d'enseignement et de recherche français ou étrangers, des laboratoires publics ou privés. 


\title{
Spectral diffusion decay of spoṇtaneous echoes in disordered systems
}

\author{
R. Maynard, R. Rammal and R. Suchail \\ Centre de Recherches sur les Très Basses Températures, C.N.R.S., BP 166 X, 38042 Grenoble Cedex, France
}

(Reçu le 13 mars 1980, accepté le 22 avril 1980)

\begin{abstract}
Résumé. - La relaxation des échos spontanés dans un système de spin $\frac{1}{2}$ ou de systèmes à 2 niveaux en intéraction est traitée dans le cadre du modèle Klauder-Anderson. L'hypothèse d'une distribution hyperbolique des temps de relaxation $T_{1}$ - ou « bruit en $1 / f »-$ conduit à une loi de relaxation très générale en $\exp (-\gamma T \tau)$ où $T$ est la température, $\tau$ l'intervalle entre les deux impulsions et $\gamma$ un coefficient ne dépendant que de l'interaction moyenne entre systèmes à 2 niveaux excités thermiquement.
\end{abstract}

\begin{abstract}
In a system of two level systems or $\frac{1}{2}$ spins, the relaxation of spontaneous echoes is obtained in the framework of the Klauder-Anderson scheme. The assumption of a hyperbolic distribution relaxation times $T_{1}$ (or " $1 / f$ noise ") leads to a general relaxation law : $\exp (-\gamma T \tau)$ where $T$ is the temperature, $\tau$ the delay time between the two pulses and $\gamma$ a coefficient depending only on the average interaction between the thermally excited two level systems.
\end{abstract}

Introduction. - It is now well established that in pure glasses low energy excitations exist which are termed tunnelling modes. These tunnelling modes are described as two level systems exhibiting an almost constant density of states for splitting energies below $1 \mathrm{~K}$. They are also strongly coupled to the phonon field and possess a very broad distribution of their $T_{1}$. In addition to these two level defects, $\mathrm{OH}$ doped glasses introduce electric dipole moments with more or less the same characteristics as the two level systems that is a uniform density of states as well as broad distribution of their $T_{1}[1,2]$. Finally, these defects interact among themselves via the phonon field rather than via the electric field [2]. Therefore in pure as well as in doped glasses a set of two level defects exists which are coupled by interactions varying as $r^{-3}$ : The dynamics of these two level systems may be treated by the same methods used for systems of true spins $S=1 / 2$ coupled by dipolar magnetic interactions. Following the scheme used by KlauderAnderson [3] for spectral diffusion in spin systems, we divide the two level systems into two classes $A$ and B : A contains the 2 level defects whose splitting energy is in resonance with the applied field-acoustic (resp. electric) for the acoustic (resp. electric) dipolar echoes. The number of $\mathrm{A}$ defects is small and largely diluted in the sample and the interactions between them may be ignored. But they are coupled [4] to the B defects via the elastic strain of the medium usually described by a hamiltonian $J_{i j} S_{z}^{i} S_{z}^{i}$ where the $S_{z}^{i}$ are the diagonal Pauli operators in the representation of the two states of the defects. Transitions between the two levels of the B defects induce fluctuating local fields at the level of the A defects. The frequency distribution of the excited packet of $A$ defects therefore becomes broader due to the dephasing effect of fluctuations of the local field. It is assumed that the system is of $T_{1}$-type : i.e. the rate of fluctuations is caused by the spin-phonon coupling. This scheme constitutes the basic model proposed first by Black and Halperin [5] for explaining the phonon echoes first discovered by Golding and Graebner [6]. However, they focus attention on the short time regime where $\tau$, the delay time between the two pulses, is shorter than the shortest thermal B spin $T_{1}$ of the $T_{1}$-distribution : In this regime they found that the decay of the echo amplitude is given by :

$$
E(2 \tau)=\exp \left(-\alpha T^{4} \tau^{2}\right)
$$

where $\alpha$ is a coefficient taking into account all the ingredients of the coupling between the 2 level defects. This regime has been studied in previous experiments by Golding and Graebner [6] and Schickfus et al. [7] but several recent experiments of Bernard et al. $[1,8]$ at lower temperatures of dielectric echoes in glasses, 
and in smoky quartz [9], indicate strongly that the decay function $E(2 \tau)$ varies as

$$
E(2 \tau)=\exp (-\gamma T \tau) .
$$

The purpose of this article is to derive in a simple way this type of variation. This law emerges in the regime where $\tau$ is inside a broad hyperbolic distribution $1 / T_{1}$ of the defects $T_{1}$. In this regime remarkably few parameters enter into the coefficient $\gamma:$ it is simply the product of the spectral and volume density of $B$ spins $N_{\mathrm{B}}$ (in $\mathrm{K}^{-1} \mathrm{~m}^{-3}$ ) and the average local dipolar field on spin A. Nothing refers to the detailed mechanism of relaxation as the one phonon assisted tunnelling motion. Neither the cut-off in the hyperbolic distribution $1 / T_{1}$ nor the details of the one-phonon process are important. We believe that this regime is much more common at very low temperature than the one described by (1), particularly if one considers the hyperbolic distribution as the $1 / f$ noise which is often invoked at low temperature (Clarke and Voss [10]).

In the Black and Halperin paper [5], this regime is qualified as " a roughly exponential decay bounded from below by $\exp \left(-\pi^{-1 / 3} m T_{1} \tau\right) »$ in their notation. However the expression and its derivation are not satisfactory because while they use the cut-off for the $T_{1}$-distribution as well as an extrapolation of the coefficient $m$ from the short time regime to this intermediate regime [11].

1. Echo amplitude. - Let us derive the expression for the spontaneous echo amplitude $E(2 \tau)$ of a collection of two level systems characterized by an energy splitting $E$ and a relaxation rate $W=1 / T_{1}$, with a density of states per unit energy and volume $N(E)$ and a joint distribution $p(W, E)$.

The two pulse echo amplitude is given by Klauder and Anderson [3] and Mims [12] :

$$
E(2 \tau)=\left\langle\left\langle\exp \left\{i \int_{0}^{2 \tau} S(t) \Delta \omega_{i}(t) \mathrm{d} t\right\}\right\rangle_{i}\right\rangle_{j}
$$

where

$$
\begin{array}{lll}
S(t)= & \text { if } & 0<t<\tau \\
S(t)=-1 & \text { if } & \tau<t<2 \tau
\end{array}
$$

and $\hbar \Delta \omega_{i}(t)=\sum_{j \in \mathrm{B}} J_{i j} S_{z}^{j}(t)$ is the time fluctuating local field on the $i$-th spin A. As $S_{z}^{j}$ is the standard Pauli matrix, we write :

$$
J_{i j}=\frac{C_{i j} \mu_{i} \mu_{j}}{R_{i j}^{3}}
$$

where $\mu_{i}$ are the dipole moments and $C_{i j}$ the coupling tensor.

$\langle\cdots\rangle_{j}$ represents the spatial average over B defects and $\langle\cdots\rangle_{i}$ the spatial average over A spins, is replaced by a time average over all histories of B spins. Since the two level systems possess two parameters $E$ and $W$, there are additional averages to be considered here.

The time average is performed through an uncorrelated sudden-jump model where the excitation flipping rate $W_{1}$ can be different from the desexcitation flipping rate $W_{2}$. In thermal equilibrium

$$
W_{1} / W_{2}=\exp \left(-E / k_{\mathrm{B}} T\right)
$$

and $W=W_{1}+W_{2}$.

This average as well as the spatial average of B spins has been calculated by $\mathrm{Hu}$ and Walker [13] using the $1 / r^{3}$ range of the interaction energy and the details of the calculation will not be repeated here. Let us write :

$$
E(2 \tau)=\exp \left\{-\frac{\pi}{6} C_{\mathrm{rms}} \mu_{\mathrm{A}} f(2 \tau)\right\}
$$

where $C_{\mathrm{rms}}=\sqrt{\left\langle C_{i j}^{2}\right\rangle}$ is an average value of the coupling constant and $\mu_{\mathrm{A}}$ another average dipole moment of A defects. Both factors define an average dipolar local field.

The Laplace transform of $f(2 \tau), F(\sigma)$ has been derived in reference [13] for $E$ and $W$ fixed (see (15) and (16) in this reference) and has the following expression

$$
\left[4 W_{1} W_{2} /\left(W_{1}+W_{2}\right)\right]\left[\left(\sigma+2 W_{1}+2 W_{2}\right)\left(\sigma+2 W_{1}\right)\left(\sigma+2 W_{2}\right) \sigma^{3}\right]^{-1 / 2}
$$

By considering the additional distribution of $E$ and $W: p(E, W)$ as well as the detailed balance of $W_{1}$ and $W_{2}$ we directly obtain :

$$
\begin{aligned}
& F(\sigma)=\iint p(E, W) \mathrm{d} E \mathrm{~d} W . \mu_{\mathrm{B}} \operatorname{sech}^{2}(E / 2 k T) \times \\
& \times 2 W\left[(\sigma+2 W)\left(\sigma+2 W /\left(1+\mathrm{e}^{-E / k T}\right)\right)\left(\sigma+2 W /\left(1+\mathrm{e}^{E / k T}\right)\right) \sigma^{3}\right]^{-1 / 2}
\end{aligned}
$$

2. The hyperbolic noise. - Suppose now that the B-two level defects have a hyperbolic distribution law for their relaxation time $T_{1}$ or in an equivalent way for their relaxation frequency $W=1 / T_{1}$ :

$$
p(W, E)=\left\{\begin{array}{l}
n(E) \frac{1}{W} \text { if } W_{\mathrm{MIN}} \leqslant W \leqslant W_{\mathrm{MAX}} \\
0 \text { elsewhere }
\end{array}\right.
$$

and a constant spectral density $n(E)=n_{0}$ for $E \leqslant E_{\mathrm{MAX}}$. 
The argument of the decay function $F(\sigma)$ has now a very simple form in the dimensionless variables $x=E / k T$ and $u=2 W / \sigma$ :

$$
\begin{aligned}
F(\sigma)=\frac{n_{0} k_{\mathrm{B}} T}{\sigma^{2}} \mu_{\mathrm{B}} \int_{0}^{E_{\mathrm{MAX} / T}} \mathrm{~d} x \operatorname{sech}^{2}( & x / 2) \times \\
& \times \int_{W_{\text {MIN } / \sigma}}^{W_{\operatorname{MAx} / \sigma}} \mathrm{d} u\left[(1+u)\left(1+u /\left(1+\mathrm{e}^{-x}\right)\right)\left(1+u /\left(1+\mathrm{e}^{x}\right)\right)\right]^{-1 / 2}
\end{aligned}
$$

At very low temperatures, $T \ll E_{\mathrm{MAX}}$ and the $\operatorname{sech}^{2}(x / 2)$ enables $x$ to be integrated to infinity. 3 different regimes now appear, defined by the value of $\sigma$ compared to the cut-off $W_{\mathrm{MAX}}$ and $W_{\mathrm{MIN}}$ :

2.1 SHORT TIME-LARGE $\sigma\left(\sigma \gg W_{\text {MAX }}\right)$ - $-u$ is always less than one since $\sigma \gg W_{\text {MAX }}$. The second integral gives $W_{\mathrm{MAX}} / \sigma$ and $F(\sigma)$ varies as $T / \sigma^{3} W_{\mathrm{MAX}}$. The inverse Laplace transform $\ln E(2 \tau)$ varies as $-\tau^{2} T W_{\text {MAX }}$. For the one-phonon assisted tunnelling relaxation $W_{\mathrm{MAx}} \approx T^{3}$ which leads to the $\tau^{2} T^{4}$ law obtained in reference [5].

2.2 LONG TIME-SMALL $\sigma\left(\sigma \ll W_{\text {MIN }}\right)$. $-u \gg 1$ and the $u$-integral can be approximated by $\sigma^{1 / 2} \operatorname{ch} x / 2$ which gives a $F(\sigma)$ varying as $\sigma^{-3 / 2}$. The inverse Laplace transform gives the usual central limit regime $\ln E(2 \tau)$ as $-\tau^{1 / 2}$ (Ref. [11]).

2.3 INTERMEDIATE REGIME $: W_{\text {MN }} \ll \sigma \ll W_{\text {MAX }}-$ The convergence of the $u$-integral is possible when $W_{\mathrm{MN}} / \sigma=0$ and $W_{\mathrm{MAX}} / \sigma=\infty$. Using

$$
\int_{0}^{\infty} \frac{\mathrm{d} x}{\operatorname{ch}^{2} \frac{x}{2}} \int_{0}^{\infty} \mathrm{d} u\left[(1+u)\left(1+\frac{u}{1+\mathrm{e}^{-x}}\right)^{\prime}\left(1+\frac{u}{1+\mathrm{e}^{x}}\right)\right]^{1 / 2}=2.635
$$

we get for $F(\sigma)$ :

$$
F(\sigma)=2.635 \frac{n_{0} k_{\mathrm{B}} T}{\sigma^{2}} \mu_{\mathrm{B}}
$$

Now the inverse Laplace transform gives for the echo amplitude :

$$
E(2 \tau)=\exp \left\{-2.635 \frac{C_{\mathrm{rms}} \mu_{\mathrm{A}} \mu_{\mathrm{B}} n_{0} k T}{h} \tau\right\} .
$$

As $n_{0} k T$ can be interpreted as the average number of excited two level systems at temperature $T$, the product $C_{\mathrm{rms}} \mu_{\mathrm{A}} \mu_{\mathrm{B}} n_{0} k T$ represents the average interaction energy among the thermally excited two level systems : it is the inhomogeneous thermal linewidth of the problem. Very striking is the disappearance of any mechanism of relaxation process in (12): this comes formally from the fact that $\tau$ is well inside the hyperbolic distribution law of relaxation times.

3. Disordered substances. - The original model of Anderson, Halperin, Varma and Philips [14] introduced two tunnelling parameters : $\Delta$ the asymmetry energy and the tunnelling coupling energy given by $\Delta_{0}=\hbar \omega_{0} \mathrm{e}^{-\lambda}$. The energy splitting is obtained by the relation $E=\left(\Delta^{2}+\Delta_{0}^{2}\right)^{1 / 2}$. It is assumed that $\Delta$ and $\lambda$ 'are uniformely distributed between two limits $\lambda_{\text {MIN }}<\lambda<\lambda_{\text {MAX }}$. The defect-lattice relaxation time $T_{1}(E, \lambda)$ is given in reference [5]

$W=T_{1}^{-1}(E, \lambda)=A E^{3} \mathrm{e}^{-2\left(\lambda-\lambda_{\mathrm{MIN}}\right)} \operatorname{coth}\left(E / 2 k_{\mathrm{B}} T\right)$.

The distribution can be rewritten in terms of the variables $E$ and $W$ :

$$
\begin{gathered}
n(E, W)=\frac{\bar{n}}{2} \frac{1}{W}\left(1-W / W_{\mathrm{MAX}}\right)^{-1 / 2} \\
W_{\mathrm{MIN}}<W<W_{\mathrm{MAX}}
\end{gathered}
$$

where $W_{\mathrm{MAX}}$ is obtained from (13) with $\lambda=\lambda_{\mathrm{MIN}}$.

In addition of this distribution law the permanent elastic dipole moment $\mu_{\mathrm{B}}$ is multiplied by the factor $\Delta / E=\left(1-W / W_{\mathrm{MAX}}\right)^{1 / 2}$. The factor $\left(1-W / W_{\mathrm{MAX}}\right)^{1 / 2}$ cancels in the product of the two expressions giving the hyperbolic distribution law (8) from which the exponential $\exp (-\gamma T \tau)$ regime is derived. These are basically the standard assumptions necessary for analysing the echo measurements on doped glasses. From the expression (12) recast in the standard formula : $E(2 \tau)=\exp \left(-2 \tau / T_{2}\right)$, the phase memory decay time is defined :

$$
T_{2}=h /\left(1.31 C_{\mathrm{rms}} \mu_{\mathrm{A}} \mu_{\mathrm{B}} k T\right)
$$


which is interpreted as the inverse average interaction frequency among the populations of the thermally excited states of two level systems. Only the order of magnitude of these parameters are known : $C_{\mathrm{rms}} \mu_{\mathrm{A}} \mu_{\mathrm{B}}=10^{-35}$ ergs $\mathrm{cm}^{3}$ corresponding to an average elastic coupling energy $M=1.5 \mathrm{eV} /$ defect (see Ref. [5]);

$n_{0}=10^{32} \mathrm{ergs}^{-1} \mathrm{~cm}^{-3}$ or $1.6 \times 10^{20} \mathrm{eV}^{-1} \mathrm{~cm}^{-3}$

from reference [15].

The product $C_{\mathrm{rms}} \mu_{\mathrm{A}} \mu_{\mathrm{B}} n_{0}$ is dimensionless and is estimated to be $10^{-3}$. From these values we get $T_{2}=10^{-5} \mathrm{~s}$ at $T=10^{-2} \mathrm{~K}$ instead of $10^{-4} \mathrm{~s}$ at $T=10^{-2} \mathrm{~K}$ from figure 4 of reference [1]. Taking into account the uncertainty of various parameters we conclude that the order of magnitude of $T_{2}$ from electric dipolar echoes is roughly described by the expression (15).

Since $T_{2}^{-1}$ is linear in temperature, the lower curve of figure 4 of reference [1] is therefore well explained. As the limits of the hyperbolic distribution of $T_{1}$ do not enter in (15), they cannot be determined. However the limit of validity of this regime is reached at lower temperatures [17] when $W_{\text {MAX }}$ is comparable to $\tau^{-1}$.

One can consider crystalline materials doped with random dipolar impurities such as $\mathrm{KCl}: \mathrm{OH}$ as a similar situation to glasses but with clustering effect of dipoles at very low temperature. This case of weak tunnelling is characterized by a distribution of tunnelling energies $\Delta_{0}$ cut-off at some low energy $\Delta_{\mathrm{c}}$ instead of ranging up to $k T\left(\Delta_{\mathrm{c}} \ll k T\right)$. This smaller value of the cut-off would reflect the collective nature of the tunnelling motion of clusters of dipoles. For these defects the noise distribution is purely hyperbolic since $W \ll W_{\mathrm{MAX}}$ and the correction to the permanent elastic dipole is also $\Delta / E \simeq 1+0\left(W / W_{\text {MAX }}\right)$ in such a way that the hyperbolic distribution is still valid. This type of weak tunnelling has been used in the analysis of the dielectric properties at very low temperature of ionic glasses BK7 [16].

4. Conclusion. - In summary, the regime of exponential decay which has been established here is based upon the assumption of a hyperbolic distribution of the relaxation time $T_{1}$ in addition to the standard ingredients of the Klauder-Anderson model namely long range $r^{-3}$ interaction, and Poissonian noise. We emphasize the generality of this regime already observed for various disordered substances at very low temperature. The hyperbolic distribution of $T_{1}$ is usually attributed to the broad distribution of potential barriers for tunnelling motion. However since the spectral power of this noise is $1 / f(f=$ frequency) the origin of this hyperbolic noise is perhaps more common than usually invoked in the context of disordered systems. Therefore the spontaneous echo decay measurement could therefore be an useful method for the investigation of this type of noise.

\section{References}

[1] Bernard, L., PichÉ, L., Schumacher, G. and Joffrin, J., J. Low Temp. Phys. 35 (1979) 411.

[2] Golding, B., v. Schickfus, M., Hunklinger, S. and Dransfeld, K., Phys. Rev. Lett. 43 (1979) 1817.

[3] Klauder, J. R., Anderson, P. W., Phys. Rev. 125 (1962) 912.

[4] Joffrin, J., Levelut, A., J. Physique 36 (1975) 811.

[5] Black, J. L., Halperin, B. I., Phys. Rev. B 16 (1977) 2879.

[6] Golding, B., Graebner, J., Phys. Rev. Lett. 37 (1976) 852 ; Phys. Rev. B 19 (1979) 964.

[7] v. Schickfus, M., Golding, B., Arnold, W., Hunklinger, S. J. Physique Colloq. 39 (1978) C6-959.

[8] Bernard, L., et al., to be published.

[9] Saint-Paul, M., et al., to be published.

[10] Voss, R. F., Clarke, J., Phys. Rev. B 13 (1976) 556.

[11] In the thesis of L. Bernard (Universite de Paris 6, 1979) the possible existence of this regime is discussed qualitatively.
[12] Mims, W. B., Phys. Rev. 168 (1968) 370.

[13] Hu, P., Walker, L. R., Phys. Rev. B 18 (1978) 1300

[14] Anderson, P. W., Halperin, B. I., Varma, C. M., Philos. Mag. 25 (1972) 1

Philips, W. A., J. Low Temp. Phys. 7 (1972) 351.

[15] Lasjaunias, J. C., RaveX, A., Vandorpe, M., Hunklinger, S., Solid State Commun. 17 (1975) 1045.

[16] Frossati, G., Maynard, R., Rammal, R., Thoulouze, D., J. Physique Lett. 38 (1977) L-439.

[17] The change towards the short regime in $\tau^{2}$ is expected when $W_{\text {MAX }}$ becomes smaller than $\sigma$. As it is generally assumed that $W_{\text {MAX }}$ decreases as $T^{3}$, the $\tau^{2}$ regime must be reached at very low temperature. This leads to an inequality for $W_{\operatorname{MAX}}(T)$ : since $T=10^{-2} \mathrm{~K}, \tau=10^{-4} \mathrm{~s}$ we are still in the intermediate regime, then $W_{\mathrm{MAX}}\left(10^{-2} \mathrm{~K}\right)>10^{4} \mathrm{~s}^{-1}$ or $W_{\mathrm{MAX}}(1 \mathrm{~K})>10^{10} \mathrm{~s}^{-1}$. 